\title{
A Multi-User Collaborative BIM-AR System to Support Design and Construction
}

James Garbett $^{1}$, Thomas Hartley ${ }^{1 \wedge}$ and David Heesom ${ }^{2 *}$

${ }^{1}$ School of Mathematics and Computer Science, Faculty of Science and Engineering, University of Wolverhampton, Wulfruna Street, Wolverhampton, WV1 1LY, UK

${ }^{2}$ School of Architecture and the Built Environment, Faculty of Science and Engineering, University of Wolverhampton, Wulfruna Street, Wolverhampton, WV1 1LY, UK

* Corresponding Author d.heesom@wlv.ac.uk

+ Orcid: https://orcid.org/0000-0003-1762-8681

^ Orcid: https://orcid.org/0000-0001-9192-3094

\begin{abstract}
Augmented Reality (AR) is fast becoming an established tool for the construction industry. Previous research reports on the conversion of BIM geometric models and the implementation of these with marker-based AR, or the use of more wide area AR taking positional input from GPS. Much of this focused on the use of AR in an individual context, so there is need to align $\mathrm{AR}$ with the more collaborative nature of BIM. By implementing marker-based AR, and connecting to a cloud-based database, the presented BIM-AR system provides the ability to view, interact and collaborate with 3D and 2D BIM data via AR with geographically dispersed teams. An Agile Scrum Method was used to develop the prototype system including a mobile AR application and a Large Touch Screen application based on and a Model, View, Controller (MVC) approach. Finally, the system was tested and verified using a focus group of construction practitioners.
\end{abstract}

Keywords: BIM, Augmented Reality, Collaboration, Cloud, Database 


\section{Introduction}

Since the phrase Augmented Reality (AR) entered the lexicon in the 1990s [1] it has been accepted as a solution that combines digitally stored and spatially referenced visual, aural or even haptic information with the physically real environment in real time [2]. Early work in the field of applying AR to the construction sector highlighted the use of see through glasses to assist in understanding the location of members in construction of a space frame, noting the potential for this in the future to become a ubiquitous technology for construction operatives [3]. Developments in the field of AR for the AEC sector continued in parallel to the development of Virtual Reality based applications and these AR developments are well documented in [4] whilst the volume of academic research in the field of AR for the construction sector was reviewed by [5] and a system of classification was proposed by [6]. With the emergence of Building Information Modelling (BIM) [7] and the more prolific use of 3D computer graphics in the design, construction and operation phases of a project, the opportunity to use the geometric information in an AR environment has been made available. In parallel to this, the rapid development of new hardware and enabling algorithms such as tracking, Visual SLAM and computer vision enhancements Ling [8] has provided the ability to exploit AR more widely. [9] proposed a framework that highlighted how BIM could be integrated with AR throughout the various stages of the project, generating a link between the digital, physical and subsequently the mixed realities.

Williams et al. [10] present a methodology to display the geometric elements within a BIM in an AR based environment. By importing a model into a tablet-based AR the user can locate themselves on an identified 'InfoSpot' location in the physical space and use the tablet to visualise the virtual model in the correct location. This is an individual based implementation and subsequently only one individual can be engaged in the simulation at one point, however it does remove the issue of internal geo-location for the AR camera. The ability to collaborate in an AR environment is seen as a critical issue to add value to the face-to-face approach [11] and 
a prototype approach to enabling this was proposed by [12] through using AR on mobile devices to facilitate discussion demonstrating that less time was required to identify problems compared to using traditional drawing media. Meža et al. [13] also demonstrated that using AR can greatly enhance the understanding of a project both for architects and engineers by a metric of $20 \%$ when compared to using a $3 \mathrm{D}$ virtual model alone. Building on this concept of greater understanding, [14] has investigated the use of AR for the development of Heritage BIM (HBIM) to help understanding of historic buildings. However, this is marker based for an individual user and whilst the use for on-site augmentation is discussed it is not realised in this specific work.

As BIM becomes the mainstream approach for construction projects, collaboration between actors in the project team is supported by new technological methods of working. To this point the use of an AR based environment to support the ability for multi-user collaboration in geographically dispersed locations has received little attention from the AEC perspective. Previous work in the field of BIM-AR has provided solutions to visualise the AR simulation in a single environment with limited ability to collaborate in that digital workspace. There is also a paucity of research surrounding collaborative AR providing the ability for multiple users to link and share information through the AR interface.

Based on the contextual backdrop provided, the aim of this study is the development of a multifaceted, multi-user, collaborative AR software platform that integrates both $2 \mathrm{D}$ and 3D data from a BIM. This aim is derived to answer the research question of whether the concept of real time, interactive, collaborative AR can provide an effective and valuable tool to support workflows within a distributed construction team. The system that is presented provides the ability to synchronously interact with other users from multiple geographic locations and share data within a BIM environment through an AR based interface. The research objectives of the project were: 
- Critically evaluate current approaches to the use of BIM with AR to establish prevailing methodologies

- Develop a proposed framework for a collaborative BIM-AR system that provided the ability for users to interact in a synchronous environment

- Implement a Scrum based software development methodology to create a software system to support user interaction across a range of platforms and user interfaces

- To evaluate the software to elicit future directions for BIM-AR and collaborative working practices

The scope of this work focused on the development of a technological solution to reuse the data derived from a BIM to support synchronous collaboration in an AR environment. Using the underlying BIM philosophy of integrating graphical and non-graphical data, the system provided the ability for the users to add data to a digital environment that could then be reintroduced to the main BIM if required.

Building on a critical review of existing literature in the field of BIM and ATR the study derived a framework for a real time, collaborative BIM-AR system. In order to implement the framework as a software prototype, an Agile software development methodology with a Scrum framework was implemented for the creation of the BIM-AR system as a multi-user visualisation and collaboration system. A modular approach was taken to the development of the AR tools and applications for the BIM-AR system were implemented in Unity, a cross-platform game engine that facilitates the creation of $2 \mathrm{D}$ and $3 \mathrm{D}$ interactive applications and games. The system was underpinned by a Client-Server database implemented in MySQL.

The remainder of this paper presents a review of existing work in the field of AR and BIM applications. This focuses on usage in design, construction and operation stages. Highlighting the gap in prevailing knowledge, a framework is presented and subsequently a system implementation is discussed to realise the framework. A testing and evaluation phase is 
considered to highlight the work undertaken by a focus group method and finally conclusions are presented and areas for future work discussed.

\section{Current Status of BIM-AR}

AR can be implemented across the full spectrum of the construction process and [15] proposes a device breakdown structure (DBS) to highlight how the technology can be used. This also subcategorises how AR could be implemented by all stakeholders from the client to the facilities manager. Previous studies have undertaken reviews of research in the field of AR for the built environment field and it is noted that it has the ability to impact all stages from planning to design, construction and operation [16]. Other studies have further sub-categorised these to highlight the specific focus of research in this field as being predominantly experimental study, case study or the development of proof of concept [5]. Recent work undertaken by [62] demonstrates that whilst AR is gaining traction within the AEC sector, the level of adoption is below that of VR and further highlights that a lower percentage of companies are expected to invest in AR than VR in the next 3 years. As such there is scope to develop tools to meet the needs of the collaborative industry to support further exploitation of AR technologies.

\section{$\underline{\text { AR for AEC Design }}$}

In the early stages of design, issues such as urban design have seen the benefits of using AR.

Katahira \& Imamura [17] proposed the use of marker based AR, viewed through a headset, to support the urban planning process, with the ability for an individual able to manipulate the 3D model using natural gestures through the Leap Motion hardware. Whilst useful, this approach is desk based and not able to contextualise the buildings in real world scale. Furthermore, the approach only provides the ability for an individual to interact with the simulation. Further studies [18] sought to resolve the problem of real world scale on a large urban models through the use of a feature map captured prior to engaging with the AR simulation 'on site'. This work 
also sought to resolve the issues around the occlusion of the real world from the virtual model [19]. A similar image tracking based approach was implemented by [20] for vision-based recognition of objects using a 3D point cloud. Similarly [21] also pre-processed additional design information using CFD, allowing the individual headset wearing user to view complex design information.

The benefit of AR is increasingly been seen as part of the suite of tools used in the architectural design process. The use of $\mathrm{AR}$ in the architectural design has also been shown to be beneficial from the point of view of understanding the aesthetics of a building within the physical environment and more effectively communicating design to clients [22]. Whilst AR could be seen as a more emerging approach, the use of Virtual Reality (VR) has been embodied into the design phase and is now being used to aid collaborative working between the teams and engagement from the client stakeholders. The use of large-scale immersive projection systems is providing the ability for a number of individuals to collaborate around a single focal point [23], whilst emerging commercially available VR based tools such as InsiteVR [24] allow collaboration within individual based VR hardware such as headsets. The benefits of these collaborative approaches now need to be brought to bear in the AR paradigm. The use of AR within design phase provides a unique approach to physically interact with the design at full scale giving a new method to critique and review design proposals at an early stage. Congruent with the fundamental concept of BIM enabling the lifecycle management of an asset, the use of AR can provide the ability for designers to understand issues around maintainability during the early phases [25]. Tang et al. [26] noted the ability for AR to span from the design phase into the construction operations which is well suited to prefabricated construction operations. The use of prefabrication will allow data stored in the design-based BIM objects to be viewed in an AR environment to assist on site assembly.

$\underline{\mathrm{AR} \text { in the construction phase }}$ 
Since some of the early work investigating the use of AR to support sequential tasks [27] the scope for AR to support construction operations has been well researched. The use for assisting with the understanding of assembly of components has received more recent attention such as [28], [29], [30]. The reporting of some of these studies has shown significant improvements to operations on the construction site, highlighting a $50 \%$ reduction in the time to complete the tasks, with fewer errors during construction. Similarly [61] have developed an AR based robot welding system which demonstrates significant temporal based improvements over traditional welding approaches using just human operators.

Work undertaken by [31] implemented image-based techniques from discrete points in time to overlay and augment the 4D model to monitor progress of the actual site compared to the developed 4D simulation. A study by [32] also investigated the integration of 4D with AR through the use of static web cameras taken from the site to create markerless registration images. As opposed to using static web camera images, [33] used images captured from UAV flights to generate markers as part of the AR development. Using UAV data [34] took a similar approach but used images captured from UAVs to generate a point cloud which could be used in the generation of geometric objects. This work then implemented on site positioning using GPS data to control camera position in a similar approach to that taken by [35] who proposed a wide area 4DAR system using GPS and mobile computing to map the camera location. Recent work by Zaher et al. [36] has moved the AR simulation towards inclusion of the 5D cost element of a project using a marker-based approach to show a 5D based video generated through Autodesk Navisworks and implemented in AR through a time sequenced 4D AR model.

More recently, AR has seen research focus on site logistics planning and the opportunity to support health and safety. [37] implemented marker-based AR to aid the understanding of the position of specific site elements on a desktop-based environment. An exhaustive review of VR and AR in the context of health and safety was undertaken by [38] and this showed that key 
areas were focused on hazard identification, training and inspection. Critical in previous studies is the functionality of the AR environment. Whilst they span a range of applications, they focus on individual use of the AR model.

\section{$\underline{\text { AR during facilities management }}$}

As the value of BIM is becoming more widely accepted within the domain of facilities management, the use of AR to visualise the BIM data during the operation phase of a built asset in seeing increasing research interest. Ammari \& Hammad [39] propose a framework that takes geometric data from a BIM model and allows the asset manager / maintenance team to view the geometric element overlaid in the physical world. Using an individual mobile device, tracking is invoked using feature recognition. Whilst the use of feature recognition is ideally suited for facilities management based AR due to the in-situ based need to view the data, this is not always possible. The approaches discussed above around pre-captured images to act as markers or occlusion may not always be possible due to the changing nature of the use of the asset. For example, in an office building, the furniture layouts may constantly change rendering previously captured images redundant. In this regard the emerging concept of spatial anchors may provide a solution to this issue in addition to the use of real time meshing [59]. Work completed by [40] and [41] propose a solution to markerless AR for FM through the use of depth sensors mounted onto mobile devices used to compare the 3D sensed data to the existing known geometry. As an alternative to this, [42] propose the use of 'natural markers' for indoor navigation in an AR environment, making use of existing fixed signage. More recent work [60] has employed iBeacon technology within a mobile application which allowed data from a BIM to be viewed in a real world contextual environment. COBie data was generated from the model and fed into a cloud database which then allowed specific fire safety equipment information to be added to the system.

\section{Issues of collaboration in AR and current research gap}


The above body of research undertaken in the field of AR for construction demonstrates the applicability of the technology for all stages of project lifecycle. Much of the research is focused on marker-based AR and arguably this is the most common and simplest form of implementation. It is noted that several studies have investigated the opportunities to move AR into a field-based application which allow for AR to be contextualised. However, throughout the range of studies much of the work is focused on the usability of the AR by an individual, providing a purely isolated engagement. This work seeks to resolve this issue whilst also addressing the issue of the need for multi-user and multi device approaches to AR \& VR as highlighted in the research agenda presented by [61]. As the AEC sector moves towards a more collaborative approach to working with the implementation of BIM processes and technologies, there exists the opportunity to exploit AR within this paradigm. Since the idea of Computer Supported Collaborative Work (CSCW) was first proposed over twenty years ago, many research papers have been published and computer systems developed that incorporate its principles to facilitating person-to-person, work-oriented, real-time communication [43], [44], [45], [46]. Communication in the context of unmediated face-to-face collaboration is driven not only by speech and conversation, but also by the use and observance of gesture, gaze and other non-verbal communication cues [44], however, when collaborators are geographically separated this non-verbal communication channel must be provided through computer-mediated means [47]. In this regard it has been suggested that to facilitate effective collaboration, the encompassing AR environment needs to nurture a sense of presence between all users as well as provide transparent mechanisms for discussing and sharing data and ideas in real time [48].

The use of AR has been deemed to aid collaboration among the project team [49]. An experimental study into communication behaviours in collaborative AR concluded that being able to see both the real world and fellow collaborators during AR sessions made for more natural patterns of communication [50]. Billinghurst and Kato [44] further explain how both the physical environment and tangible objects within it support collaboration during spatially 
referenced work activities, such as design and planning, by providing, for example, a reference frame for task-centred communication. Studies by [47] concur that enabling AR users to generate their own references within the environment is a critical factor for the success of collaborative environments. They also acknowledge the difficulties encountered in creating effective interaction metaphors for collaborative VEs, noting however that when collaborating in $\mathrm{AR}$, a user will need system features that support communication and mutual awareness between participants, while further recommending the facilitation of methods for pointing or indicating at both real and virtual content [47].

Dong et al [51] proposed a prototype tool ARVita which allowed multiple users to collaborate in a single location through a marker-based AR simulation. Whilst all co-located, each user was engaged in the session through an individual headset. To further enhance this concept, [52] sought to integrate AR with telepresence to provide a higher level of collaborative working. This work provided a prototype solution that allowed 3 individuals to visualise an AR based model whilst also providing teleconferencing facilities between the participants. This was a fundamental aspect as in addition to the technological based solution it also provided social trust between the participants. In a study by Chen et al [58] the issue of collaborating in different geographic environments was resolved by allowing remote operatives to view a live video stream of AR environment. Whilst this approach provides a more passive interaction between multiple users it does allow a synchronous connection between participants. It does not, however, provide the ability for multiple users to interact and collaborate in their own AR space whilst in different locations.

From a BIM perspective, it is clear that in addition to the need to share the visual aspect of a model through the AR environment, there is also a need to share further information and collaborate to update the 'I' element of a BIM. A significant body of previous work in the field of BIM-AR has provided solutions to visualise the AR simulation in a single environment with limited ability to collaborate. Where AR has been used as a tool to support collaboration, this 
has been focused on users co-existing in the same physical space whilst interacting with the AR model. Subsequently, work in the field of collaborative AR has not yet fully exploited the ability for multiple users to link and share information through the AR interface whilst in geographically dispersed locations. Furthermore, there is a paucity of work that has fully exploited the potential to allow multiple users to synchronously interact with the AR model to add information and have this available in real time to other users within the simulation environment. This study identifies these limitations as a gap in current knowledge and critical element that needs addressing in order to develop a truly collaborative AR system that can support the BIM process and workflow. It is this aspect that is the focus of this study to develop a tool to allow geographically dispersed team members to interact through a cloud based visual and informational dataset using locally an AR visualisation.

\section{Proposed approach}

Based on the above prevailing work in the field of BIM and AR, an approach is developed to meet the aim of developing a multi-user collaborative AR based tool that could be used at various stages of design and construction.

Specifically, the system should provide;

- $\quad$ Real time, multi-user interaction through AR

Previous work has highlighted that much of the work in AR is focused on stand along applications, whilst the sector increasingly requires multiple actors to engage in the design data through BIM processes. Subsequently, there is need to allow multiple users to synchronously interact.

- Real time bi-directional synchronous information transfer As the BIM process becomes more prolific and refined, there exists a need to transfer information between stakeholders in a synchronous manner. Hitherto, there has been a 
limited amount of work undertaken to allow real time data transfer to take place through an AR environment.

- Ability to add, and spatially locate, non-graphical data through both 2D and 3D data By its very nature, the AEC sector utilises spatial data during all phases of the lifecycle. The ability to geolocate data is a fundamental aspect of BIM tools, however this capability does not yet appear in the BIM-AR paradigm. This work seeks to align the application of BIM-AR such that it can function on parity with prevailing BIM and VR platforms.

- Integration of 3D BIM data in AR, through the implementation of mobile devices The industry now works significantly with mobile devices, and there is a need for any new tools to work across multi users and multiple devices [62].

- The use of a multiple approaches to AR implementation including analogue marker and large screen collaborative touch devices

As AR is advancing in respect to its technological development, there are multiple methods to position augmented objects in the real world setting. Furthermore, marker based AR provides a method to visualise the environment in a stable position. As large scale devices are becoming more prominent in the workplace, there exists the need to develop a tool which can support both analogue and digital methods of viewing the AR environment through this medium.

CSCW has been shown to aid collaboration, whilst the use of AR to visualise the geometric environment provides a greater understanding of the design. Building on the issues discussed above, the proposed approach would also need to enable users to generate information which can be spatially located and instantly shared with other users through the AR interface. This bidirectional ability to communicate synchronously coupled with the ability to interact with the AR environment and engage with real time data sharing, instantly sharing with other users in their own version of the AR tool is critical to the success, and a unique element in this approach. In order to achieve the above, the underlying proposed framework for BIM-AR tool is proposed 
in Figure 1. A Client-Server approach will allow multiple users to engage with the same dataset in a synchronous manner, whilst this will also support the development of an online information database which can act as a repository for data added to the design model by multiple users. The server side will also control how data is linked to specific locations on the $2 \mathrm{D}$ and $3 \mathrm{D}$ geometric BIM data in the simulation.

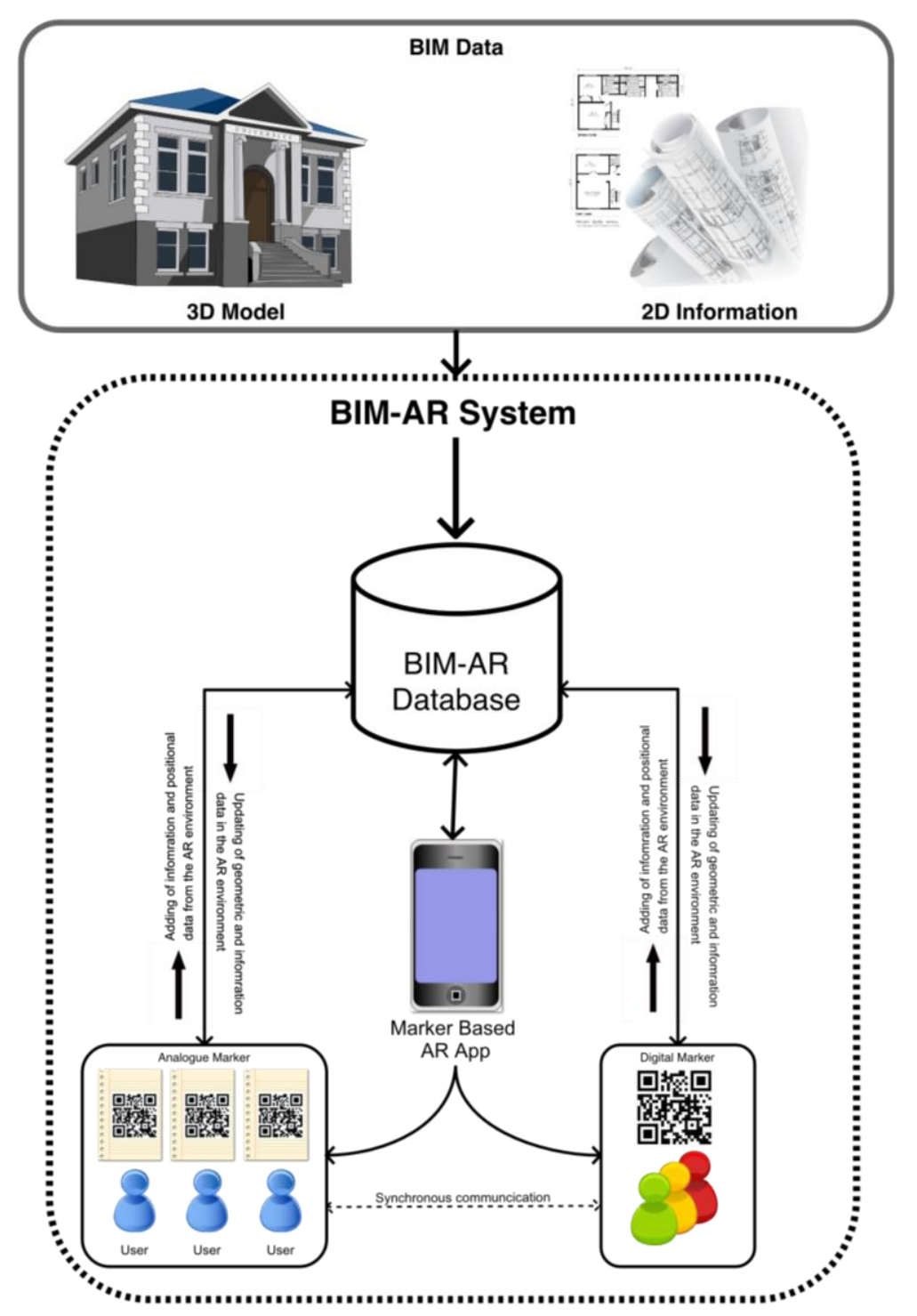

Figure 1: Proposed Framework for BIM-AR 
Building on the proposed approach to the system, an Agile software development methodology with a Scrum framework was implemented for the creation of the BIM-AR system as a multiuser visualisation and collaboration system. The Agile scrum methodology breaks up software development into small increments that involve a complete software development cycle (e.g. planning, design, coding and testing). Once an increment has been created it can be demonstrated to stakeholders for feedback with future increments then integrating the feedback into development. This development methodology was selected because it focuses on fast delivery of the software in complete functional increments. It also gave frequent opportunity for the work to be evaluated and changes made throughout the development of the project (Figure 2).

The review of existing literature in the paradigm of BIM/Construction AR developments provided the basis for the functional requirements within the proposed framework. These then informed the first development sprint which focused on the creation of a marker-based AR application. A marker-based AR approach was selected as this allowed rapid development and also opened up the potential for the tool to be utilised across multiple platforms using ubiquitous mobile devices. In addition, as the use of larger touch screen collaborative workspaces such as touch screen tables are becoming more prominent [53] then the ability to operate the BIM-AR tool on this medium is also considered.

The developed system had clear user views of data (e.g. AR application) and clear data sets (e.g. 3D models, 2D plans and user annotations); therefore, a Model, View, Controller (MVC) approach was taken for the software architecture. The MVC approach meant that separate representations of data could be isolated and presented to the user in different ways. The database (e.g. user annotations) was stored separately on a MYSQL database hosted on a server, and the view (e.g. user interface) was implemented for a mobile AR application and a large table-top touchscreen application. 


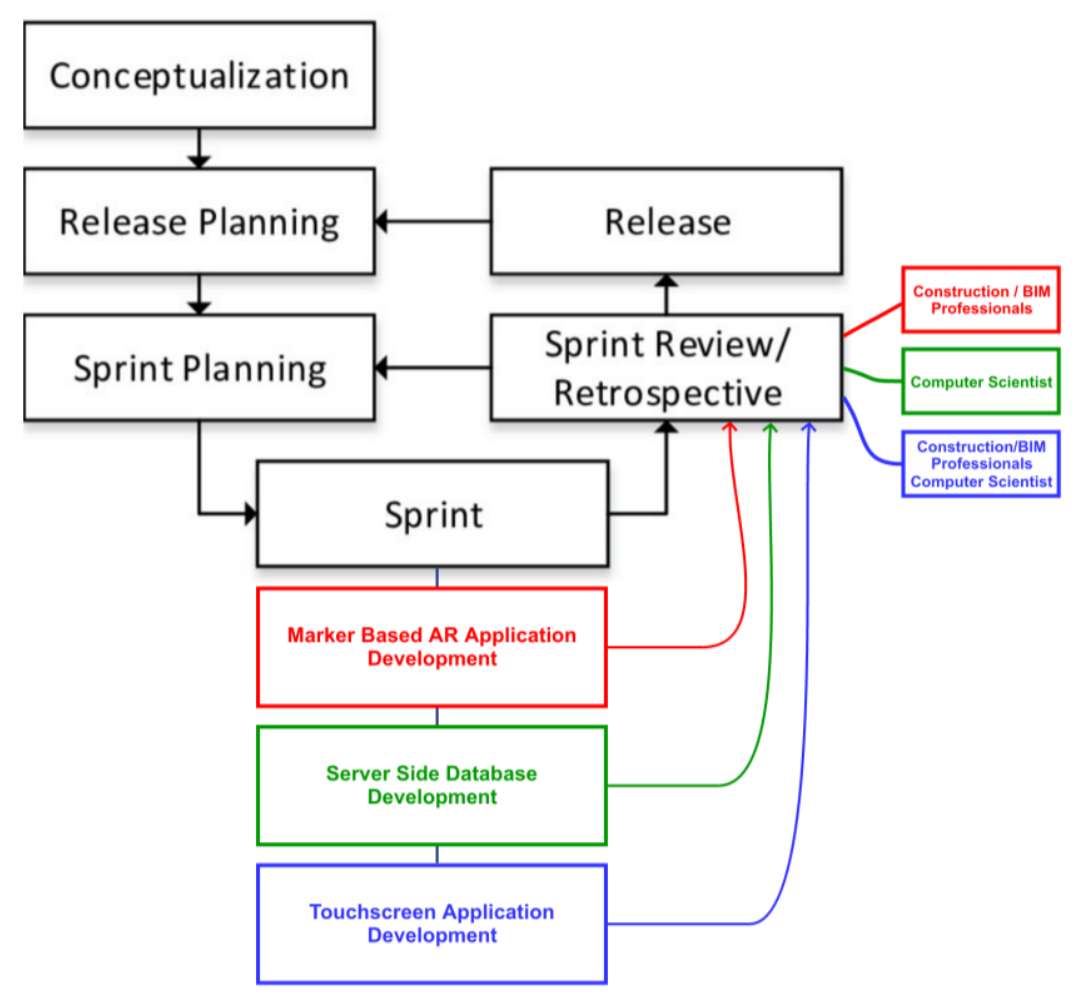

Figure 2: Scrum approach for BIM-AR development

(Adapted from: Lin et al [54])

The first Scrum sprint increment focused on the development of a marker-based AR application implemented on a mobile device. The following Scrum sprint then developed a server-side database which connected to the AR application and provided the ability to read/write data to the user interface. Following the developments achieved in these first two sprints, reviews were then undertaken by three individuals, two of these were construction/BIM focused and a further was a computer science professional. From a construction perspective, the reviewers included a BIM professional with over 5 years experience and an architect with 10 years experience. The feedback from these focused on the usability and functionality requirements of the BIM-AR system whilst the review and feedback from the computer science practitioner focused on the user interface and backend functions of the tool. 
Feedback from the reviews during the first two sprints were used to update requirements for the third sprint which focused on the development of a standalone application which could operate on a large touchscreen. The touchscreen application would provide the ability to i) provide the ability to view $2 \mathrm{D}$ data including architectural plans in a digital medium and ii) provide digital markers for visualising the 3D BIM via an AR application. The user can interact with the digital 2D drawings to add markers to pick a location on the plan and add annotation information. By clicking on the location, the position in the $\mathrm{x}-\mathrm{y}$ plane on the model is determined and the $\mathrm{z}$ elevation of the marker can then be specified according to the building level to give a 3dimensional point in space for the annotation. This positional data and the annotation information is then written to the server-side database and the 3D marker can then be viewed in a 3D AR environment.

\section{System Implementation}

As discussed above, the BIM-AR system is developed using a Scrum approach and subsequently the development focused on a modular basis (Figure 3). The aim of the system was to provide the ability to visualise, interact and add information to a BIM through an AR medium. All applications for the BIM-AR system were implemented in Unity, a cross-platform game engine that facilitates the creation of 2D and 3D interactive applications and games. Unity is a mature and popular game engine, especially on mobile platforms, that has been used to create many interactive applications and games; therefore, it is the ideal tool for the development of our BIM-AR multi-user visualisation and collaboration system. All code for the applications was written in C\# and we made use of two key plugins to facilitate development. The initial process was to convert a BIM database (3D and 2D information) into the BIM-AR system. This followed the approach as noted by [55] for the transfer of 3D model data from 
Autodesk Revit to Unity using FBX. 2D plan based data was exported as PDF and imported into the developed Unity application.

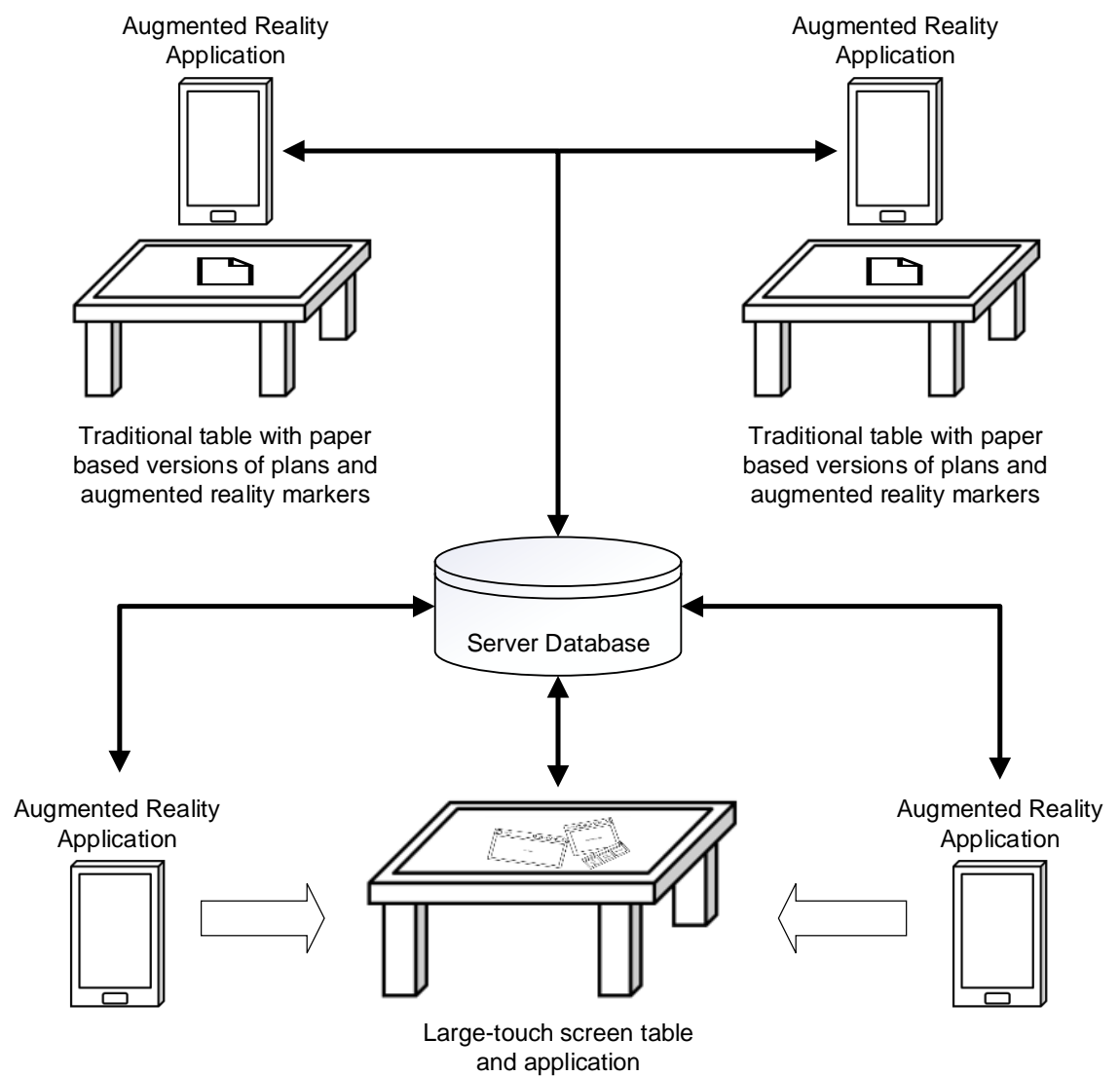

Figure 3: BIM-AR System architecture

BIM-AR: Augmented Reality Application (ARA)

There are currently a range of software development toolkits (SDK) (e.g. Vuforia, Wikitude and ARToolKit) available to support the generation of AR simulations [56]. Apple and Google have also recently developed AR SDKs that are tightly integrated with their mobile operating systems and hardware. Apple and Google's SDKs have the potential to offer more robust features and better tracking results due to their integration with mobile software and hardware; however, their benefits may not be portable across to other mobile systems. For this work the Vuforia SDK was used to develop the ARA as it is currently a widely deployed AR SDK, 
offering cross-platform support and robust AR features, such as tracking multiple images targets and 3D object tracking in real time. Therefore, it is an ideal solution that offers the greatest range of platform support. In addition, the TouchScript plug-in was used to add support for standard touchscreen gestures (e.g. pinch to zoom) to our AR applications.

A Marker based approach to the ARA was used as it provided the ability to generate markers on either physical based objects (i.e. paper plans) or digitial based sources (i.e. large multi touch devices). This flexibility in data source allows the ARA to work in many environments where technology may be limited. In addition, marker based augmented reality allows multiple models to be easily associated with a single piece of data (e.g. an architectural plan) and more easily visualised because the marker is easier to recognise than an image and it is easier to position and rotate to suit the environment lighting and multiple users (Figure 4).
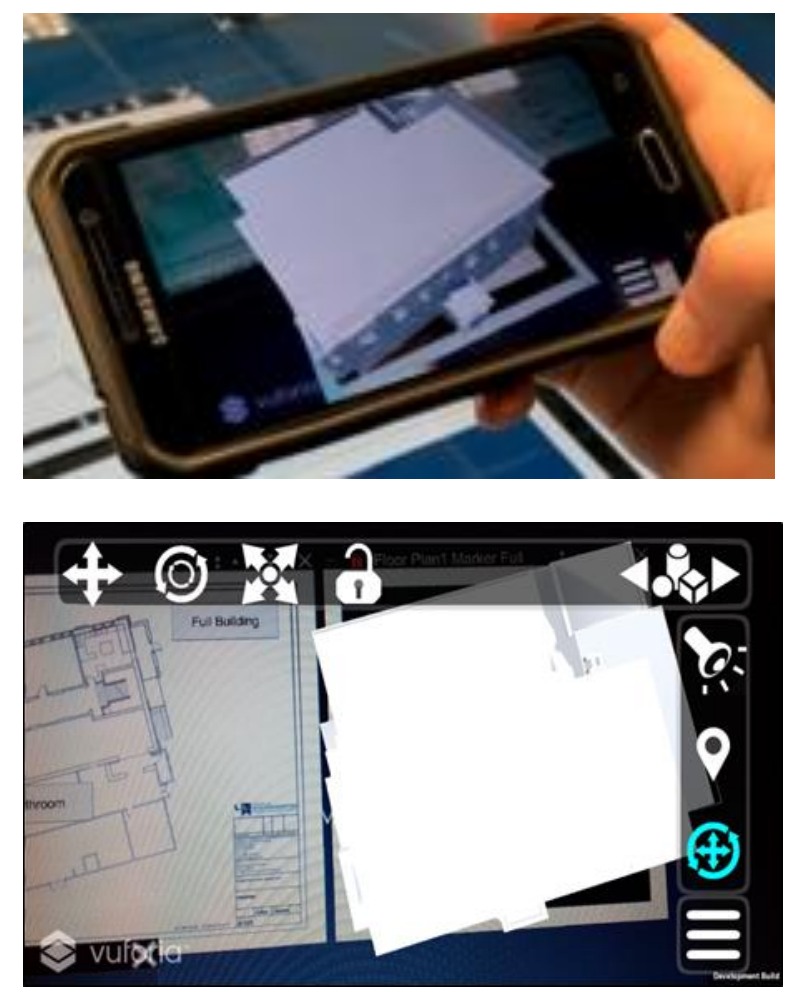

Figure 4: BIM-AR system - mobile AR interface.

The ARA incorporated many features to support collaboration between users. For example, the 3D model could be manipulated in multiple ways; including the ability to change the location, 
rotation and scale of the 3D data without affecting the marker itself. This feature allows more than one person to independently use the same marker to view 3D data. To make the changes to their view of the 3D data the user simply adjusts sliders. Three sliders are used for each option allowing the user to rotate and translate in a cardinal plane or direction. The user can also lock the $3 \mathrm{D}$ data to the screen, so it is no longer dependant on the marker. This feature can be toggled on and off and is useful when the user wants to maintain a specific view of the data. Additional navigation features were also built into the ARA including giving users access to the directional light, text annotation and orientation sub-menus. The annotation menu displays options for annotations, including colour, text input and the ability to visually add a marker to the 3D data. The orientation menu displays the options for the model to be manipulated such as translation, rotation and scale. When a menu item is selected a further menu is displayed across the top of the screen (Figure 4). The clipping tool slices the model along a cutting plane and allows the interior of a model to be viewer.

One of the key collaboration features in the BIM-AR system is the ability for users to add annotations to 3D data and have these available during synchronous sessions to communicate changes and updates in real time. The annotations allow for communication between all users of the BIM-AR application and touchscreen-table application. To add a marker a user needs to tap the 3D data at the location where they want to add the point of interest. When the user taps the 3D data an icon is added; the user is then able to add text to the annotation by using an onscreen keyboard. The user also assigns a colour to the annotation; the colour could represent a type of comment, for example these could be linked to specific members of the design/construction team. The user receives conformation when the annotation has been sent to the server (Figure 5). The mechanism for sending data to the online databased is discussed further in the following section. 


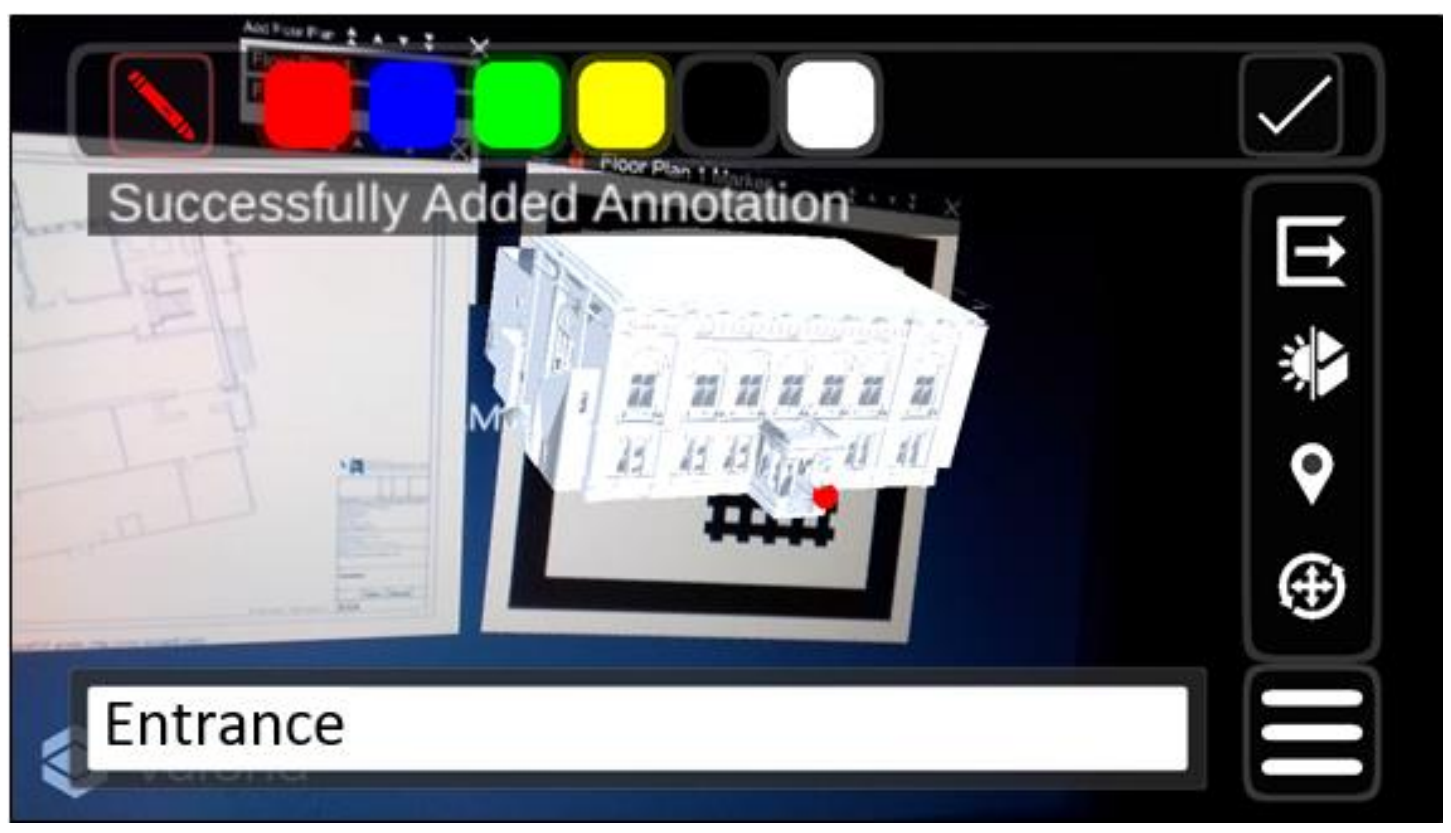

Figure 5: Synchronous data addition to the AR model

\section{BIM-AR: Client-Server Database (CSD) Development}

To store and manage all the data added by users during a session of the BIM-AR system, a MySQL database was implemented. The key table in the database stores user annotations based on a session ID. Each annotation was stored as a row in the database table. Each row included an annotation ID, colour, location, the model and sub-model to which the annotation was attached, annotation text and session ID. The database is hosted on a server, whilst the mechanism used to add data to the database utilised the sending of a HTTP POST request to a PHP script, which in turn talked to the online MySQL database. The php script takes parameters including the text, coordinates of the marker, colour, session name and flag. The parameters are then added to the database. Both the ARA and touchscreen applications fetch data from the database using a php script, which returns a string that contains all the annotations stored in the database for the session. The database is called regularly to keep the information up to date.

When the annotations are refreshed the new annotations are shown. The annotations have a set of coordinates associated with them. When the annotations are drawn, they are mapped onto the 
2D data to give an accurate representation of where the marker was added on the 2D plans imported into the BIM-AR system.

A final key collaboration feature in the BIM-AR system is the ability to create user sessions. This feature allows multiple groups of users to work with data at the same time. The session allows a user to connect with a specific set of information on the database. When users are logged into the same session on either application, any annotations added will be displayed on the ARA and the Large Touch Screen Application (LTSA) and data is synchronously transferred between the users (Figure 6)

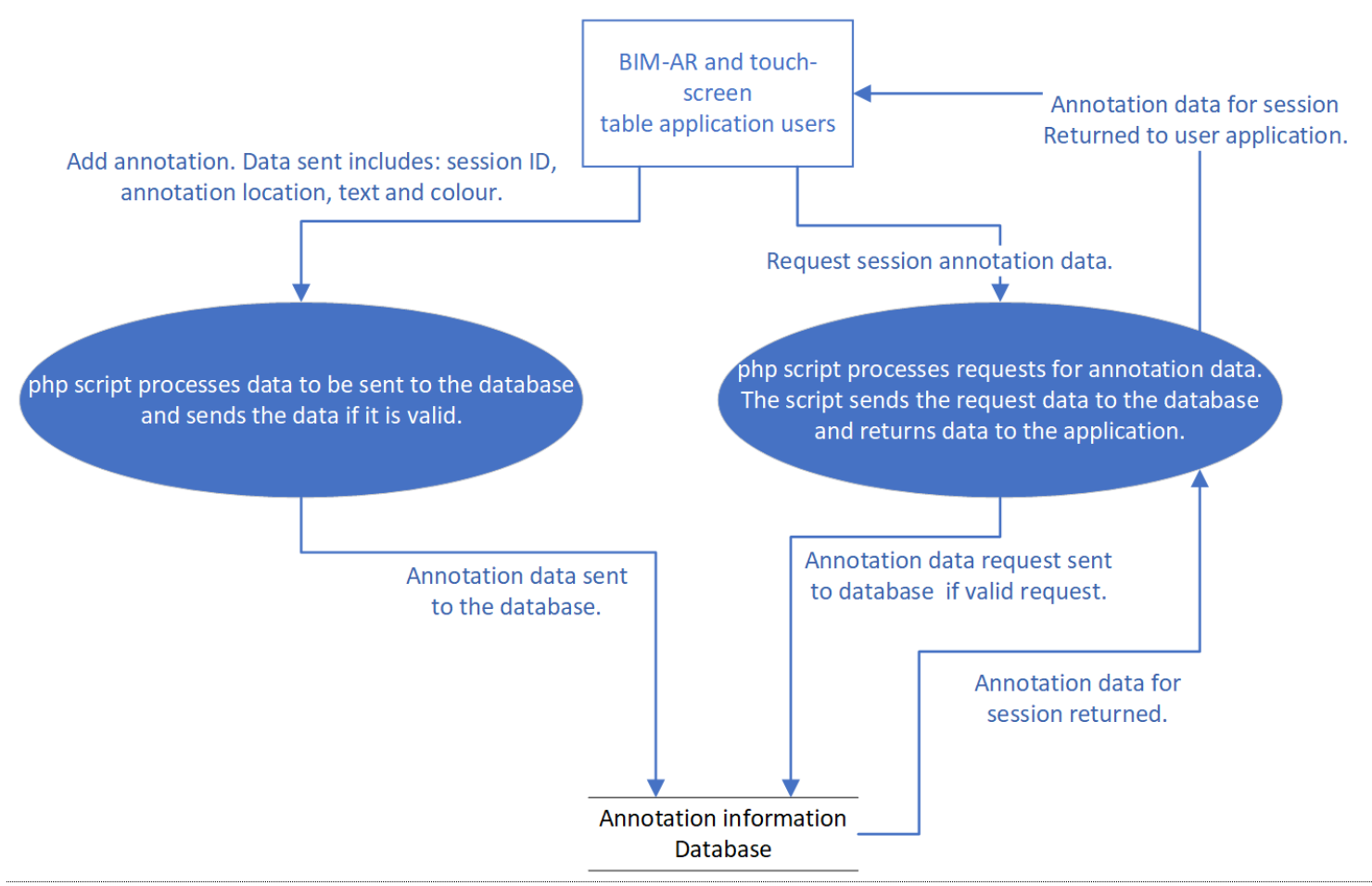

Figure 6: Data flow between components of the BIM-AR system

Sessions in the BIM-AR system are created in either the ARA or the LTSA and Figure 7 highlights the data input screen for creating or joining a session. Either of the applications will show all sessions that are available on the database and the user can pick anyone of the open 
sessions to log into. If there are no sessions the user can either create a new session or still use the application without having access to the collaborative features.

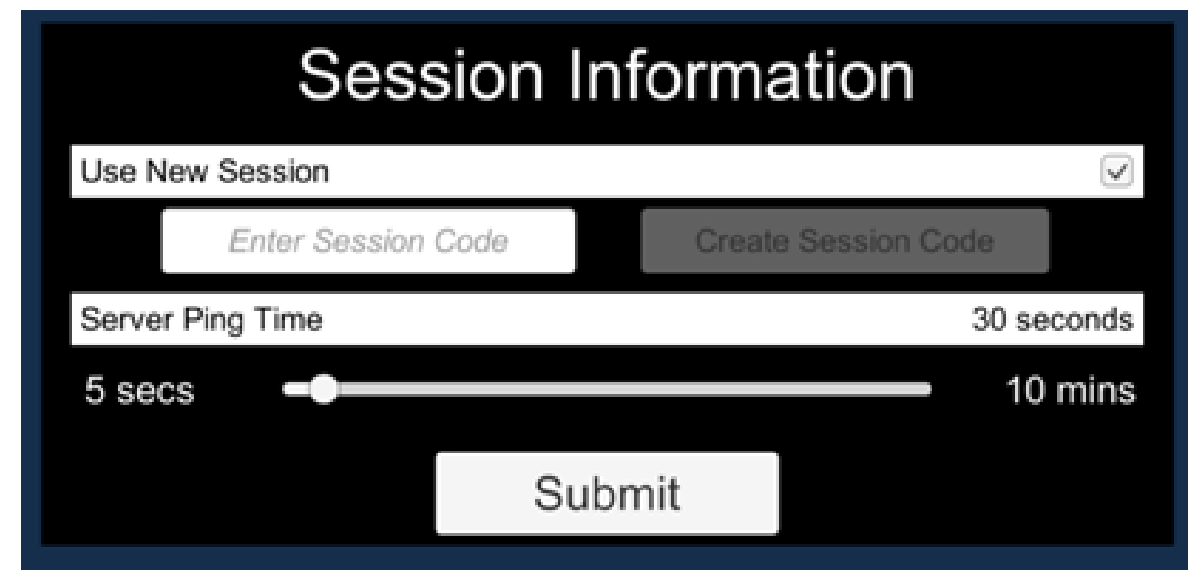

Figure 7: The session creation menu

\section{BIM-AR: Large Touch-Screen Application (LTSA)}

The large touch-screen application (LTSA) component of the BIM-AR system is designed to display multiple instances of data, such as plans and elevations, and allow multiple users to collaborate by being able to simultaneously manipulate screen objects via a touch interface. The application is also implemented in Unity version 5.6.2F1 and coded using C\#. The application incorporates the ability to display either one single AR marker which can be viewed by multiple users working around the large touch screen or multiple AR markers allowing multiple users of the large touchscreen to each visualise 3D models individually through the single large touchscreen interface. Users can switch markers on and off by touching an icon that is overlaid on the 2D data. When AR markers are produced and displayed on the large touch screen interface, the previously developed ARA can be used to visualise the 3D data as discussed previously. Using the ARA with the LTSA provides the ability to interact in the same way as if paper-based markers were used. However, the LTSA has additional functionality to support further collaboration in the BIM-AR system. 
Interaction with the large touch-screen table application is achieved using standard touchscreen gestures, for example pinch is used to zoom in and out of a plan. However, gestures have been adapted for the large touch-screen display. This approach to the interface design facilitates a collaborative user experience by allowing multiple users to interact with different or identical elements of the data at the same time (Figure 8).

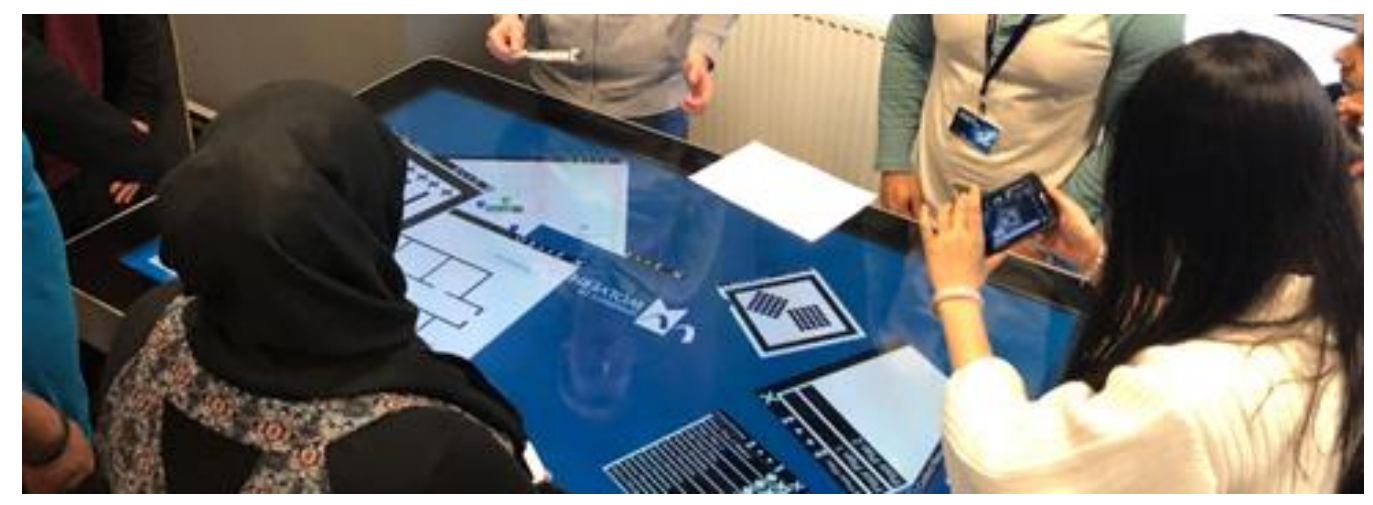

Figure 8: Multiple users collaborating using the LTSA in a single session.

The range of 2D data within the BIM-AR system can be added to the LTSA via a list menu system. Each element of 2D data has a toolbar and dropdown menu system that allows features to be switch on/off or added. For example, each element of 2D data has a dropdown menu that allows augmented reality markers and text annotations to be shown or hidden. Using the ARA individual annotations and information can be added through the 3D AR model, however using the LTSA, markers can be added to the 2D plan information and this is then added to the database and can subsequently be visualised in the 3D AR environment using any of the BIMAR interfaces. As previously discussed with the ARA, the annotations are shown with a colour tag to identify the annotation (Figure 9). 


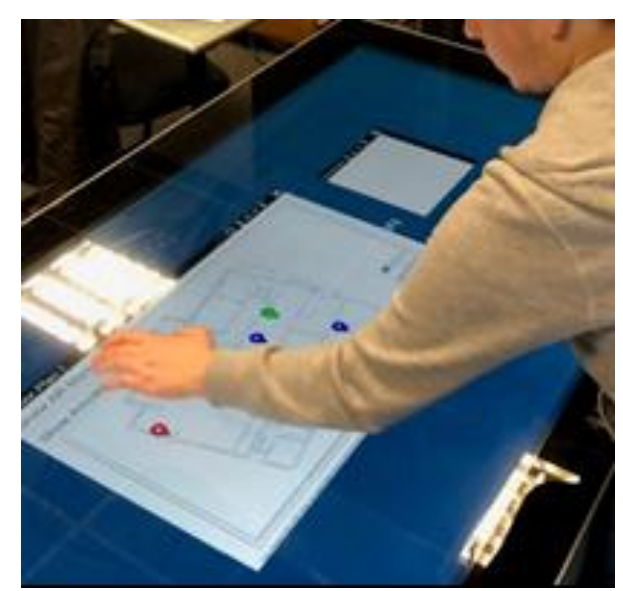

Figure 9: Adding information to the BIM-AR database using 2D plans in the LTSA

By clicking on the location on the $2 \mathrm{D}$ plan allows the user to add annotation information to the BIM-AR database. The marker position records $x-y$ plane location on the model and the $z$ elevation of the marker can then be specified according to the building level to give a 3dimensional point. The 2D plans are taken from the BIM software tools and converted to be held in the LTSA application. In the current form the annotation information is purely written to the BIM-AR database, however future work will seek to link this database back to the BIM application such that the information added by the users can be imported back into the BIM authoring tool. The ability to add and visualise information to specific points on the model by either using 3D AR or the 2D plans provided flexibility for users to engage with the system in multiple ways. This ensured collaboration could take place through either medium using either 2D or 3D data derived from the BIM.

\section{Testing and Evaluation}

As discussed, the Scrum methodology was used for the software development and testing. Feedback was elicited at the end of each of the Scrum sprints and following the final development of all aspects of the BIM-AR system a focus group was held with Eleven individuals to demonstrate the system and derive feedback on the approach taken and potential 
future avenues of enhancement. A focus group approach was taken because it offers a fast and cost-effective way to obtain qualitative insights and feedback from practitioners [57]. In a software engineering context, it allows us to elicit rich, qualitative information and potentially reveal insights that are difficult to capture with other methods [57].

The focus group was comprised of Architects, Architectural Technologists, Civil Engineers and Construction Managers which gave a representative sample of potential users of the system across the various stakeholders within the AEC sector. Furthermore, a range of years of experience in the sector gave more confidence in responses and how these could be related to prevailing working practices (Table 1).

\begin{tabular}{|l|l|l|}
\hline Participant & Industry Role & Industry Experience (years) \\
\hline P1 & Architectural Technologist & 5 \\
\hline P2 & Architect & 10 \\
\hline P3 & Architect & 6 \\
\hline P4 & Civil Engineer & 3 \\
\hline P5 & Architectural Technologist & 5 \\
\hline P6 & Construction Manager & 8 \\
\hline P7 & $\begin{array}{l}\text { Postgraduate Researcher (Construction } \\
\text { Manager) }\end{array}$ & 3 \\
\hline P8 & Architect & 9 \\
\hline P9 & Interior Architect & 7 \\
\hline P10 & Construction Manager & 4 \\
\hline P11 & Architectural Technologist & 2 \\
\hline
\end{tabular}

Table 1: Focus Group participants

In addition to the focus group evaluating the system from a user perspective, a technical evaluation was undertaken on the performance of the software prototype. In order to ensure a smooth simulation and user experience, the system was required to run in real-time at more than 60 frames per second. Benchmark tests during operation with test models showed an average frame rate during operation of over this threshold when using the ARA on Android (Samsung Galaxy S7 hardware) and iOS (Apple iPad Air 2 hardware). In addition, a test was run on the access time of the MYSQL database from the ARA and the LTSA. In testing, these 
demonstrated a low latency access time of less than $5 \mathrm{~ms}$ in each of the test cases. Updates from the database back to other users AR simulations also took less than 1 second in each test case.

The implementation of the AR tracking was also tested and Vuforia SDK has been shown to have consistently good tracking accuracy at a range of angles and depth to markers under ambient lighting conditions [63]. During testing, the ARA was used and pointed towards the image targets from a range of angles and positions. During these tests the ARA recognised image targets within less than 1 second. A minimum physical target size of $12 \mathrm{~cm}$ square was used in order to support good quality recognition and in using these the 3D model displayed no latency within the system.

The methodology employed for the operation of the focus group session commenced with an interactive demonstration of the system with all participants having the ability to download the ARA and interact with both paper-based markers and then also the LTSA. A collaborative session was established to allow all participants to fully engage with the synchronous abilities of the system within respect to data input and sharing. Once the demonstration was completed, a semi-structured open forum was undertaken and moderated by the development team. The questions in the semi structured interview were themed (Table 2). Initially, at the start of each theme during the focus group an initial question was asked to ascertain the general feeling of the group. From here, further qualitative responses were elicited to provided deeper feedback on the system.

\begin{tabular}{|l|l|}
\hline Question Groupings & Initial Question \\
\hline Previous experience of using AR & $\begin{array}{l}\text { Have you had previous experience of using } \\
\text { AR? }\end{array}$ \\
\hline Ease / intuitive use of use of the system & Did you think the system was easy to use? \\
\hline $\begin{array}{l}\text { Ability of the system to collaborate with } \\
\text { others }\end{array}$ & $\begin{array}{l}\text { Do you think the system can support } \\
\text { collaborative working? }\end{array}$ \\
\hline $\begin{array}{l}\text { Potential use of the system for the AEC } \\
\text { sector }\end{array}$ & $\begin{array}{l}\text { Do you think the system has potential to } \\
\text { support the AEC sector? }\end{array}$ \\
\hline
\end{tabular}

Table 2: Focus Group question themes and initial quantitative questions 
The respondents highlighted that a small number had experience of using AR $(n=3)$, however this had been in the field of gaming and marketing. They had not had experience of using AR tools within a professional setting.

All participants $(\mathrm{n}=11)$ highlighted that the system was intuitive to work with and the user interface on the modules was simple to use. In addition, all participants did not experience any detection or tracking performance issues and they did not report any noticeable unstable / jittery tracking or difficulty in detecting a target which could have impacted the perception of system usability.

Most of the participants $(\mathrm{n}=10)$ deemed that the premise of the BIM-AR system was a success in respect to supporting collaboration and noted that it provided ' ...instant collaboration. People in different geographical locations can work in AR and get instant feedback'. Another participant noted that this system would improve communication between stakeholders, 'To explain where a certain wall is on a building may take you half an hour over the phone but if you can drop that pin on it and it takes them straight to it, it'll save everybody time'. This feature also gives the client the ability to discuss aspects of the design with a degree of accuracy without having to be in the same room. In addition, as the application does not destroy information this will also mean the client could leave feedback at any time of the day as the architect does not have to be there.

There was broad consensus that the tool had potential to support the AEC sector $(n=10)$ and AR had significant potential in the future. In terms of usage, the group identified design team and construction team meetings as a major potential use of the technology. The ability to visualise in AR was more 'intuitive' than screen-based 3D; however, it was suggested that rather than mobile devices the system could be ported to emerging holographic technologies. In addition, it was suggested that the focus of this application should be targeted towards the interactions between the client and the industry worker (e.g. architect). This might be tailored by having a client specific setting where their annotation can be clearly separated from that of the architects. 
Currently, this can be achieved by using differently coloured tags. The participants also suggested that it would be useful to zoom into the interior of a building and turn off walls. This would allow clients to add annotation within the model rather than the exterior and help with interior design.

Some identified the potential of the system to be used in an education setting for the teaching of students and the potential to interact with others in other countries through AR. The LTSA functionality of having the ability to have multiple markers for individuals was seen as beneficial with one participant noting the benefit of '.. being able to have my own view and rotate the model without having to walk around the table'. However, some did question how many operational sites would have large touch screen devices available and this was seen as a potential future development for the industry.

\section{Conclusions and Future Work}

Collaborative methods of working is becoming critical to the success of the construction sector. BIM is supporting the move to this collaborative method of working and exchanging digital data. Augmented Reality (AR) is now becoming more widespread across many genres, and the AEC sector is also embracing this. AR has been the focus of a range of research studies and these have ranged from investigating more collaborative approaches to enhancing the visualisation of 3D data using AR. Whilst many system and studies have purported the use of collaborative VR systems and the abilities to interact synchronously with 3D data in a VR environment, there has been relatively little with respect to this approach in AR.

The BIM-AR system proposed in this paper has sought to address some aspects of this gap. Using an Agile Scrum software development methodology, the study developed a system which allowed 3D and 2D BIM data to be incorporated into an AR platform which could then be interacted with synchronously using paper or digital marker-based AR. During the AR session, 
information could be added to the AR environment and stored in an online database which could be accessed by any of the users instantaneously. The system was then evaluated by a focus group of construction professionals who identified the potential benefits of the tool.

The research question identified in this study focused on understanding whether the concept of real time, interactive, collaborative AR can provide an effective and valuable tool to support workflows within a distributed construction team. Through the implementation and feedback from the focus group it has been highlighted the tool not only provided a mechanism to support collaboration through instantaneous feedback but that the approach of using AR provided a unique visualisation experience that allowed an intuitive view of the model within the users own real world environment.

Future work will seek to further develop and enhance this BIM-AR platform in respect to emerging hardware thus removing the need for AR to be viewed through mobile devices. In addition, the data stored in the database is presently divorced from the initial BIM dataset and so future developments will provide a mechanism to reintegrate data from the BIM-AR system back into the original BIM database.

\section{References}

[1] T.P. Caudell, D.W. Mizell

Augmented reality: an application of heads-up display technology to manual manufacturing processes.

Twenty-Fifth Hawaii International Conference on System Sciences, IEEE (1992), pp. 659-669 10.1109/HICSS.1992.183317 
[2] R.T. Azuma

A survey of augmented reality.

Presence: Teleoperators \& Virtual Environments, 6 (4) (1997), pp 355-385,

$\underline{10.1162 / \text { pres.1997.6.4.355 }}$

[3] A. Webster, S. Feiner, B MacIntyre, W. Massie, T. Krueger

Augmented reality in architectural construction, inspection and renovation.

Third Congress on Computing in Civil Engineering, ASCE (1996), pp. 913-919, 10.1.1.30.477

[4] H. Chi, S. Kang, X. Wang

Research trends and opportunities of augmented reality applications in architecture, engineering, and construction.

Automation in Construction, 33, (2013), pp.116-122, 10.1016/j.autcon.2012.12.017

[5] S. Rankohi, L. Waugh

Review and analysis of augmented reality literature for construction industry.

Visualization in Engineering, 1(9), (2013), 10.1186/2213-7459-1-9

[6] X. Wang, M.J. Kim, P.E. Love, S. Kang

Augmented reality in built environment: Classification and implications for future research.

Automation in Construction, 32, (2013), pp.1-13, 10.1016/j.autcon.2012.11.021

[7] C. Eastman, P. Teicholz, R. Sacks, K. Liston

BIM Handbook: A Guide to Building Information Modeling for Owners, Managers, Designers, Engineers and Contractors 
$2^{\text {nd }}$ ed. John Wiley \& Sons, New York, 2011 978-0470541371

[8] H. Ling

Augmented Reality in Reality.

IEEE Multimedia, 24(3), (2017), pp. 10-15, 10.1109/MMUL.2017.3051517

[9] X. Wang, P.E. Love, M.J. Kim, C. Park, C. Sing, L. Hou

A conceptual framework for integrating building information modeling with augmented reality.

Automation in Construction, 34, (2013), pp.37-44, 10.1016/j.autcon.2012.10.012

[10] G. Williams, M. Gheisari, P. Chen, J. Irizarry

BIM2MAR: An efficient BIM translation to mobile augmented reality applications.

Journal of Management in Engineering, 31(1), (2015), 10.1061/(ASCE)ME.1943-5479.0000315

[11] S. Lukosch, M. Billinghurst, L. Alem, K. Kiyokawa

Collaboration in augmented reality.

Computer Supported Cooperative Work (CSCW), 24(6), (2015) pp.515-525, 10.1007/s10606-

$\underline{015-9239-0}$

[12] T. Lin, C. Liu, M. Tsai, S. Kang,

Using augmented reality in a multiscreen environment for construction discussion.

Journal of Computing in Civil Engineering, 29 (2014). 10.1061/(ASCE)CP.1943-5487.0000420

[13] S. Meža, Ž Turk, M. Dolenc

Measuring the potential of augmented reality in civil engineering.

Advanced Engineering Software, 90 (2015) pp.1-10. 10.1016/j.advengsoft.2015.06.005 
[14] L. Barazzetti, F. Banfi

Historic BIM for mobile VR/AR applications, in: Anonymous Mixed Reality and Gamification for Cultural Heritage

Springer, 2017, pp. 271-290. ISBN: 978-3-319-49607-8

[15] K. Lee, S. Kwon, T. Ko, Y. Kim

A Study AR Based Smart Device for Work Management at Plant Construction Sites. International Symposium on Automation and Robotics in Construction, 35, (2018), pp926-932, $\underline{10.22260 / \text { ISARC2018/0129 }}$

[16] J. Ren, Y. Liu, Z. Ruan

Architecture in an age of augmented reality: applications and practices for mobile intelligence BIM-based AR in the entire lifecycle International Conference on Electronic Information Technology and Intellectualization (ICEITI), (2016), pp664-674 ISBN: 978-1-60595-364-9

[17] T. Katahira, H. Imamura

Development of a city planning simulation system using leap motion in the AR space. International Journal of New Computer Architectures and Their Applications, 6 (2016) pp. 150$156,10.17781 / \mathrm{P} 002236$

[18] L. Carozza, D. Tingdahl, F. Bosché, L. Van Gool Markerless vision-based augmented reality for urban planning Computer-Aided Civil and Infrastructure Engineering, 29 (2014) pp. 2-17, 10.1111/j.1467$\underline{8667.2012 .00798 . x}$ 
[19] L. Carozza, E. Valero, F. Bosché, G. Banfill, R. Mall, M. Nguyen

UrbanPlanAR: BIM mobile visualisation in urban environments with occlusion-aware augmented reality

Lean and Computing in Construction Congress - Joint Conference on Computing in Construction, (2017), pp. 229-236, 10.24928/jc3-2017/0152

[20] H. Bae, M. Golparvar-Fard, J. White

High-precision vision-based mobile augmented reality system for context-aware architectural, engineering, construction and facility management (AEC/FM) applications Visualization in Engineering, 1(3), (2013), 10.1186/2213-7459-1-3

[21] T. Fukuda, K. Mori, J. Imaizumi

Integration of CFD, VR, AR and BIM for Design Feedback in a Design Process eCAADe Conference 33, pp.665-672

[22] J. Wang, X. Wang, W. Shou, B. Xu

Integrating BIM and augmented reality for interactive architectural visualisation Construction Innovation, 14 (2014) pp.453-476, 10.1108/CI-03-2014-0019

[23] A. Fenais, N. Smilovsky, S.T. Ariaratnam, S.K. Ayer

A meta-analysis of augmented reality challenges in the underground utility construction industry

Construction Research Congress 2018: Infrastructure and Facility Management, (CRC 2018), ASCE, pp.80-89, 10.1061/9780784481295.009

[24] InsiteVR. [viewed May 24, 2019]. Available from: https://www.insitevr.com. 
[25] I.A. Khalek, J.M. Chalhoub, S.K. Ayer

Augmented Reality for Identifying Maintainability Concerns during Design

Advances in Civil Engineering, (2019), 10.1155/2019/8547928.

[26] S. Tang, L. Tang, Z. Wu, W. Zheng, C. Chen

A conceptual workflow for BIM based prefabrication design visualization with augmented reality.

16th International Conference on Construction Applications of Virtual Reality, (2016), pp. 376386.

[27] D. Reiners, D. Stricker, G. Klinker, S. Müller

Augmented reality for construction tasks: Doorlock assembly.

Proceedings of the international workshop on Augmented reality: placing artificial objects in real scenes, (1998), pp. 31-46, 10.1.1.3.1687

[28] M. Funk, A. Bächler, L. Bächler, T. Kosch, T. Heidenreich, A. Schmidt

Working with augmented reality: A long-term analysis of in-situ instructions at the assembly workplace

10th International Conference on Pervasive Technologies Related to Assistive Environments, (2017), pp.222-229, $10.1145 / 3056540.3056548$

[29] L. Hou, X. Wang, M. Truijens

Using augmented reality to facilitate piping assembly: an experiment-based evaluation Journal of Computing in Civil Engineering, 29(1), (2013), 10.1061/(ASCE)CP.1943$\underline{5487.0000344}$

[30] A.R.M. Cuperschmid, M.G. Grachet, M.M. Fabrício 
Development of an Augmented Reality environment for the assembly of a precast woodframe wall using the BIM model

Ambiente Construído. 16 (2016) pp.63-78, 10.1590/s1678-86212016000400105

[31] M. Golparvar-Fard, F. Peña-Mora, S. Savarese

D4AR-a 4-dimensional augmented reality model for automating construction progress monitoring data collection, processing and communication Journal of Information Technology in Construction (ITCon), 14, (2009), pp.129-153

[32] H. Kim, L. Kang

AR-based 4D CAD system using marker and markerless recognition method Procedia engineering, 196 (2017) pp. 29-35, 10.1016/j.proeng.2017.07.169

[33] M. Wen, S. Kang

Augmented reality and unmanned aerial vehicle assist in construction management Computing in Civil and Building Engineering, (2014), pp.1570-1577, $\underline{10.1061 / 9780784413616.195}$

[34] S. Zollmann, C. Hoppe, S. Kluckner, C. Poglitsch, H. Bischof, G. Reitmayr Augmented reality for construction site monitoring and documentation Proceedings of the IEEE, 102(2), pp.137-154, 10.1109/JPROC.2013.2294314

[35] N. Moore, D. Heesom

Integrating Interactive 4D CAD with Wide-Area Augmented Reality to enhance On-Site Construction Planning.

International Conference on Construction Applications of Virtual Reality, (2011), pp. 484-495 
[36] M. Zaher, D. Greenwood, M. Marzouk

Mobile augmented reality applications for construction projects

Construction Innovation,18(2), (2018),pp. 152-166, 10.1108/CI-02-2017-0013

[37] A.R. Singh, V.S.K. Delhi

User behaviour in AR-BIM-based site layout planning

International Journal of Product Lifecycle Management, 11(3), (2018), pp. 221-244,

$\underline{\text { 10.1504/IJPLM.2018.094715 }}$

[38] X. Li, W. Yi, H. Chi, X. Wang, A.P. Chan

A critical review of virtual and augmented reality (VR/AR) applications in construction safety

Automation in Construction, 86, (2018), pp. 150-162, 10.1016/j.autcon.2017.11.003

[39] K.E. Ammari, A. Hammad

Collaborative BIM-based markerless mixed reality framework for facilities maintenance Computing in Civil and Building Engineering, (2014), pp. 657-664,

$\underline{10.1061 / 9780784413616.082}$

[40] M. Kopsida, I. Brilakis

Markerless BIM registration for mobile augmented reality based inspection

Proceedings of the International Conference on Smart Infrastructure and Construction, (2016), pp. $1631-1636$

[41] M. Kopsida, I. Brilakis

BIM Registration Methods for Mobile Augmented Reality-Based Inspection 
11th European Conference on Product \& Process Modelling eWork and eBusiness in Architecture, Engineering and Construction, (2016), 10.17863/CAM.42274

[42] C. Koch, M. Neges, M. König, M. Abramovici

Natural markers for augmented reality-based indoor navigation and facility maintenance Automation in Construction, 48, (2014), pp.18-30, 10.1016/j.autcon.2014.08.009

[43] M. Billinghurst, S. Weghorst, T. Furness

Shared space: An augmented reality approach for computer supported collaborative work.

Virtual Reality, 3(1), 25-36, 10.1007/BF01409795

[44] M. Billinghurst, H. Kato

Collaborative augmented reality.

Communications of the ACM, 45(7), (2002), pp. 64-70, 10.1145/514236.514265

[45] J. Jiang, S. Zhang, Y. Li, M. Shi, M.

CoFrame: A framework for CSCW applications based on grid and web services.

IEEE International Conference on Web Services (ICWS'05), IEEE, 10.1109/ICWS.2005.33

[46] W. Zhou, D. Heesom, P. Georgakis

CSCW design for 4D construction planning in a distributed real-time VR environment. 7th International Postgraduate Research Conference in the Built and Human Environment, pp. $202-211$

[47] J.W. Chastine, K. Nagel, Y. Zhu, L. Yearsovich 
Understanding the design space of referencing in collaborative augmented reality environments.

Proceedings of Graphics Interface, (2007), pp. 207-214, 10.1145/1268517.1268552

[48] K. Muramoto, M. Jemtrud, S. Kumar, B. Balakrishnan, D. Wiley

Emerging technologies in a tele-collaborative design studio between the pennsylvania state university and carleton university.

Journal of Information Technology in Construction (ITcon), 13(41), pp. 660-673.

[49] A. Cardoso, do Santos Peres, Isabela Cristina, E. Lamounier, G. Lima, M. Miranda, I.

Moraes

Associating Holography Techniques with BIM Practices for Electrical Substation Design. International Conference on Applied Human Factors and Ergonomics, (2017), pp. 37-47, 10.1007/978-3-319-60204-2_5

[50] K. Kiyokawa, M. Billinghurst, S.E. Hayes, A. Gupta, Y. Sannohe, H. Kato, H.

Communication behaviors of co-located users in collaborative AR interfaces.

International Symposium on Mixed and Augmented Reality, IEEE, (2002),

10.1109/ISMAR.2002.1115083

[51] S. Dong, A.H. Behzadan, F. Chen, V.R. Kamat

Collaborative visualization of engineering processes using tabletop augmented reality Advances in Engineering Software, 55 (2013), pp. 45-55, 10.1016/j.advengsoft.2012.09.001

[52] X. Wang, P.E. Love, M.J. Kim, W. Wang

Mutual awareness in collaborative design: An Augmented Reality integrated telepresence system. 
Computers in Industry, 65(2), (2014), pp. 314-324, 10.1016/j.compind.2013.11.012

[53] G. Schubert, D. Schattel, M. Tonnis, G. Klinker, F. Petzold

Tangible mixed reality on-site: interactive augmented visualisations from architectural working models in urban design.

In: Celani G., Sperling D., Franco J. (eds) Computer-Aided Architectural Design Futures.

The Next City - New Technologies and the Future of the Built Environment. CAAD Futures 2015. Communications in Computer and Information Science, vol 527, 10.1007/978-3-662-

47386-3_4

[54] J. Lin, H. Yu, Z. Shen, C. Miao

Studying task allocation decisions of novice agile teams with data from agile project management tools.

International conference on Automated software engineering, ACM/IEEE, (2014), pp. 689-694, $\underline{10.1145 / 2642937.2642959}$

[55] Y. Liu, G. Tanudjaja, Z. Jiang, N. Beck

Workflow of Exporting Revit Models to Unity, State College, Pennsylvania

https://bim.wikispaces.com/file/view/Revit_3DS_Unity+Workflow.pdf, Accessed 27th Jun 2018

[56] D. Amin, S. Govilkar

Comparative study of augmented reality SDKs

International Journal on Computational Science \& Applications. 5(1), (2015),pp. 11-26, 10.5121/ijcsa.2015.5102

[57] J. Kontio, L. Lehtola, J. Bragge. 
Using the Focus Group Method in Software Engineering: Obtaining Practitioner and User Experiences.

International Symposium on Empirical Software Engineering (ISESE '04). IEEE, (2004), pp. 271-280, 10.1109/ISESE.2004.35.

[58] K. Chen, C.T. Li, J.C. Cheng

A BIM-based location aware AR collaborative framework for facility maintenance management.

Journal of Information Technology in Construction (ITcon), 24(19), (2019), pp.360-380.

[59] Microsoft.

Azure Spatial Anchors, Microsoft Azure.

https://azure.microsoft.com/en-gb/services/spatial-anchors/ Accessed Oct 23, 2019

[60] Y. Chen, Y. Lai, Y. Lin

BIM-based augmented reality inspection and maintenance of fire safety equipment Automation in Construction, 110 (2020), 10.1016/j.autcon.2019.103041

[61] P. Tavares, C.M. Costa, L. Rocha, P. Malaca, P. Costa, A.P. Moreira, A. Sousa, G. Veiga Collaborative Welding System using BIM for Robotic Reprogramming and Spatial Augmented Reality.

Automation in Construction, 106, (2019), 10.1016/j.autcon.2019.04.020

[62] J. Delgado, L. Oyedele, P. Demian, T. Beach

A research agenda for augmented and virtual reality in architecture, engineering and construction 
Advanced Engineering Informatics, 45, (2020), 10.1016/j.aei.2020.101122

[63] G. Kiss, C. Palmer, H Torp

Patient Adapted Augmented Reality System for Real-time Echocardiographic applications

Augmented Environments for Computer-Assisted Interventions. AE-CAI 2015. Lecture Notes in Computer Science, vol 9365. Springer, Cham. 10.1007/978-3-319-24601-7_15 